\title{
Alzheimer's disease and the microbiome
}

\section{Surjyadipta Bhattacharjee and Walter J. Lukiw*}

Departments of Neurology, Neuroscience and Ophthalmology, LSU Neuroscience Center, Louisiana State University Health Sciences Center,

New Orleans, LA, USA

${ }^{*}$ Correspondence: wlukiw@lsuhsc.edu

Edited by:

Ludovic Martin, Université de Nantes, France

Reviewed by:

Steven R. Brenner, Saint Louis University, USA

Keywords: Alzheimer's disease, evolution, gastrointestinal, innate immune response and inflammation, Metchnikoff, neuro-inflammation, probiotics, symbiosis

\begin{abstract}
"Microbial colonization of mammals is an evolution-driven process that modulates host physiology, many of which are associated with immunity and nutrient intake"-Heijtz et al. (2011)
\end{abstract}

The recognition of the human microbiome (HM) as a substantial contributor to nutrition, health and disease is a relatively recent one, and currently, peerreviewed studies linking alterations in microbiota to the etiopathology of human disease are few. Emerging studies indicate that the HM may contribute to the regulation of multiple neuro-chemical and neuro-metabolic pathways through a complex series of highly interactive and symbiotic host-microbiome signaling systems that mechanistically interconnect the gastrointestinal (GI) tract, skin, liver, and other organs with the central nervous system (CNS). For example, the human GI tract, containing $95 \%$ of the HM, harbors a genetically diverse microbial population that plays major roles in nutrition, digestion, neurotrophism, inflammation, growth, immunity and protection against foreign pathogens (Forsythe et al., 2012; Collins et al., 2013; Douglas-Escobar et al., 2013; see below). It has been estimated that about 100 trillion bacteria from up to 1000 distinct bacterial species co-inhabit the human GI tract, albeit in different stoichiometries amongst individuals, and the varying combinations and strains of bacterial species amongst human populations might contribute, in part, to "humanbiochemical" or "genetic-individuality" and resistance to disease (Aziz et al., 2013; Lukiw, 2013). Interestingly, HM participation in human physiology may also help explain the genome-complexity conundrum-for example why the 26,600 protein-encoding transcripts in Homo sapiens are far fewer in number, than for example, the rice genome (Oryza sativa; which has about 46,000 functional genes). One thousand different strains of bacteria might be expected to contribute up to $4 \times 10^{6}$ potential mRNAs to the human transcriptome, thus making the human host-plus-microbiome genetic complexity closer to $4,026,600 \mathrm{mRNA}$ transcripts, and a clear "winner" of human genetic complexity over that of rice and other species (Venter et al., 2001; Foster and McVey Neufeld, 2013; Lukiw, 2013). The very recent observation of microbiomederived small non-coding RNA (sncRNA) and micro RNA (miRNA) translocation and signaling across endothelial barriers, between cells and tissues, and even perhaps between individual species indicates that human neurobiology may be significantly impacted by the actions of HMmediated sncRNA or miRNA trafficking, and the integration of a cell, tissue or an entire organism into its local environment (Zhao et al., 2006; Alexandrov et al., 2012; Sarkies and Miska, 2013; Reijerkerk et al., 2013; unpublished). This opinion paper encompasses what we know concerning the contribution of the HM to neurological disease, with specific emphasis on Alzheimer's disease (AD) wherever possible.

Firstly, the microbiome of the human GI tract is the largest reservoir of microbes in the body, containing about $10^{14}$ microorganisms; over $99 \%$ of microbiota in the gut are anaerobic bacteria, with fungi, protozoa, archaebacteria and other microorganisms making up the remainder. There is currently an expanding interest in the ability of intestinal bacteria to influence neuro-immune functions well beyond the GI tract. Since mitochondria are believed to originate from bacteria via endosymbiotic relationships that formed very early in the evolutionary history of eukaryotes, cross-reactivity of mitochondria and immunological responses to intestinal bacterial constituents could have deleterious effects on mitochondrial function through some form of molecular mimicry; this is partially evidenced by the inflammatory basal ganglia disorder Sydenham's chorea, rheumatic fever and the link to the facultative anaerobe Streptococcus (Carrasco-Pozo et al., 2012; Douglas-Escobar et al., 2013; Hayashi, 2013; Hornig, 2013 see below). Established pathways of GI-CNS communication currently include the autonomic nervous system (ANS), the enteric nervous system (ENS), the neuroendocrine system, and the immune system (Camfield et al., 2011; Heijtz et al., 2011; Forsythe et al., 2012; Aziz et al., 2013; Collins et al., 2013; Foster and McVey Neufeld, 2013; Schwartz and Boles, 2013). Stress further influences the composition of the HM, and reciprocal communication between the CNS and the HM also influences stress reactivity (Forsythe et al., 2012; Foster and McVey Neufeld, 2013). Surprisingly, neuronal signaling pathways along the bidirectional GI-CNS axis remain poorly understood despite their important roles: (i) in coordinating metabolic- and nutritivefunctions, and (ii) in their functional disruption in chronic diseases such as metabolic syndrome, diabetes, obesity, anxiety, autoimmune-disease and stressinduced neuropsychiatric disease (Lukiw and Bazan, 2006; Bravo et al., 2012; Foster and McVey Neufeld, 2013; Hornig, 2013; Udit and Gautron, 2013). Studies of the ENS in "germ-free" mice, i.e., those 
missing their microbiome, indicates that commensal intestinal microbiota are absolutely essential for passive membrane characteristics, action potentials within the ENS, and the excitability of sensory neurons, thus providing a potential mechanistic link for the initial exchange of signaling information between the GI tract microbiome and the CNS (Foster and McVey Neufeld, 2013; Hornig, 2013; McVey Neufeld et al., 2013). Indeed, secretory products of the GI microbiome and translocation of these signaling molecules via the lymphatic and systemic circulation throughout the CNS are just beginning to be identified. For instance, the GI tract-abundant gram-positive facultative anaerobic or microaerophilic Lactobacillus, and other Bifidobacterium species, are capable of metabolizing glutamate to produce gamma-amino butyric acid (GABA), the major inhibitory neurotransmitter in the CNS; dysfunctions in GABA-signaling are linked to anxiety, depression, defects in synaptogenesis, and cognitive impairment including $\mathrm{AD}$ (Aziz et al., 2013; Hornig, 2013; Mitew et al., 2013; Paula-Lima et al., 2013; Saulnier et al., 2013). To cite another important example, brain-derived neurotrophic factor (BDNF) has pleiotropic effects on neuronal development, differentiation, synaptogenesis and the synaptic plasticity that underlies circuit formation and cognitive function, and has been found to be decreased in brains and serum from patients with schizophrenia, anxiety and AD (Carlino et al., 2013; Lu et al., 2013; Mitew et al., 2013). In experimental infection models known to lead to alterations in the microbiota profile, BDNF expression was found to be reduced in the hippocampus and cortex of "germ free" mice, and this reduction in the expression of BDNF was found to associate with increased anxiety behavior and progressive cognitive dysfunction (Carlino et al., 2013; Foster and McVey Neufeld, 2013; Lu et al., 2013).

Equally interesting are microbiome interactions with the N-methyl-Daspartate (NMDA) glutamate receptor, a prominent CNS device that regulates synaptic plasticity and cognition (Lakhan et al., 2013). For example, the NMDA-, glutamatetargeting, glutathione-depleting and oxidative-stress-inducing neurotoxin
$\beta$-N-methylamino-L-alanine (BMAA), found elevated in the brains of patients with amyotrophic-lateral sclerosis (ALS), Parkinson-dementia (PD) complex of Guam and $\mathrm{AD}$, has been hypothesized to be generated by cyanobacteria of the intestinal microbiome, and stress, GI tract disease or malnutrition may further induce BMAA abundance to ultimately contribute to neurological dysfunction (Brenner, 2013). Other HM-resident cyanobacteria-generated neurotoxins such as saxitoxin and anatoxin- $\alpha$ may further contribute to human neurological disease, especially during aging when the intestinal epithelial barrier of the GI tract becomes more permeable (Tran and Greenwood-Van Meerveld, 2013). Interestingly, BMAA, a neurotoxic amino acid not normally incorporated into protein, has been linked with intra-neuronal protein misfolding, a hallmark feature of the amyloid peptide-enriched senile plaque lesions, and resultant inflammatory neurodegeneration, that characterize $\mathrm{PD}, \mathrm{AD}$ and prion disease $(\mathrm{He}$ and Balling, 2013; Hornig, 2013; Mulligan and Chakrabartty, 2013; Schwartz and Boles, 2013). Hence, besides potentially altering CNS neurochemistry and neurotransmission, HM-bacteria not only secrete molecules that potentially modulate systemic- and CNS-amyloidosis, they also widely utilize their own amyloid peptides as structural materials, adhesion molecules, toxins, molecules that function in the protection against host defenses and auto-immunity. The specific contribution of host bacteria and bacterial amyloid, however, to misfolding, amyloidogenic diseases such as $\mathrm{AD}$ remain to be more clearly defined (Schwartz and Boles, 2013). The HM further appears to condition host immunity to foreign microbes, including viral infection and xenobiotics, while regulating autoimmune responses that can impact homeostatic metabolic- and neural-signaling functions within the CNS (Ball et al., 2013; Douglas-Escobar et al., 2013; Hornig, 2013). An increased prevalence of autoimmunity, exposure to pathogens both pre- and post-natally, and findings of anti-brain antibodies, common in disorders as diverse as anxiety, schizophrenia, obsessivecompulsive disorder, depression and autism, together suggest that differences in exposure and genetic vulnerability toward HM-mediated autoimmunity may be significant determinants of age-related neurological disease course and outcome (Ball et al., 2013; Douglas-Escobar et al., 2013; Hornig, 2013).

Regarding potentially pathogenic micobiota stationed outside of the GI tract, about $95 \%$ of all humans harbor the highly neurotrophic herpes simplex-1 (HSV-1) in their trigeminal ganglia, but whether this is a neutral or symbiotic relationship, or detrimental to the host, remains open to speculation. Induction of HSV-1, and other forms of endogenous viral reactivation are certainly stressrelated, but whether GI tract HM-derived metabolites are involved in these kinds of pathogenic activation mechanisms is not well understood (Hill et al., 2009; Prasad et al., 2012). Recent studies suggest that activation of endogenous HSV1 or other neurotrophic microorganisms, including host-embedded prions, are intimately linked to neurological stressors linked to amyloidogensis, inflammatory neurodegeneration and progressive cognitive impairment, and may be a contributor to the early development of, or predisposition to, schizophrenia and $\mathrm{AD}$ (Hill et al., 2009; Prasad et al., 2012; Ball et al., 2013; Manuelidis, 2013). Indeed, correlation of metabolic and neurological phenotypes with the GI tract HM and other specific endogenous bacterial or viral profiles derived from independent molecular analytical technologies should be increasingly useful for deciphering complex host-microbiome relationships in healthy human brain aging and in neuropsychiatric disease (Xie et al., 2013).

Further studies of symbiotic HM-CNS communication intrinsically suggests the potential for microbial-based therapeutic strategies that may aid in the augmentation of the HM, for the treatment of human disease, including neurological disorders (Forsythe et al., 2012; Collins et al., 2013). The original observation of the health-promoting benefits of GI tract bacteria and the HM was first introduced in 1907 by the Russian biologist Ilya Metchnikoff (Nobel Prize in Medicine 1908, shared with Paul Erlich; Buryachkovskaya et al., 2013). Metchnifoff's works focused on prokarytoic immunology, phagocytosis, the 
anti-aging properties instilled by host bacteria, inflammation as a protective adaptation against injury, and early ideas on neurogastroenterology (Buryachkovskaya et al., 2013; Saulnier et al., 2013). Hence, for well over 100 years, host-beneficial GI tract bacteria, collectively known as probiotics, have been proposed to be useful to human health, and more recently have been added to various foods and diets because of their positive health-promoting effects (Singh et al., 2013). The beneficial actions of bacterial-based probiotics are highly inter-related, and are thought to function, in part: (i) to aid in complex carbohydrate fermentation and absorption; (ii) to provide a significant source of a range of essential vitamins, particularly those of the vitamin $\mathrm{B}$ and $\mathrm{K}$ group; (iii) to compete with pathogenic microorganisms in the GI tract; (iv) to antagonize and neutralize enteric pathogens; (v) to metabolize and neutralize dietary carcinogens; and (vi) to favorably modulate the host's immune response to resist infection and disease. Besides the potential application of probiotics in the prevention and treatment of various health conditions and diseases such as allergies, GI and urogenital tract infections, inflammatory disease, cystic-fibrosis and certain cancers there is increasing interest of positive microbiome effects toward the CNS via neural, neuroendocrine, neuroimmune and humoral links (Duncan and Flint, 2013; He and Balling, 2013; Saulnier et al., 2013). For example, there is preliminary research on the influence of probiotics and nutritional factors on the prognosis of multiple sclerosis (von Geldern and Mowry, 2012), cognition (Camfield et al., 2011), neurogastroenterology in general (Saulnier et al., 2013), and stress-related psychiatric conditions including anxiety, autism, depression and schizophrenia (Bravo et al., 2012; Prasad et al., 2012; Douglas-Escobar et al., 2013). Advances in probiotic technologies in CNS disease research are already raising a number of ethical, legal, and socioeconomic concerns (Slashinski et al., 2012).

Lastly, the US NIH "Roadmap" program has recently initiated the HM project (HMP; http://commonfund.nih.gov/ $\mathrm{Hmp} /$ ), using recently discovered genomic technologies with the specific aims (i) to characterize the microbial communities at several different sites on the human body, including nasal, oral and otic cavities, the skin, GI and urogenital tracts; (ii) to analyze the role of these microbes in homeostatic human physiology; (iii) to catalog specific microbiome speciation, composition and correlation with disease; and (iv) to generate resources enabling comprehensive characterization of the HM by multiple independent research groups. These investigations present a highly significant and exciting avenue for future study, and suggest new and perhaps unconventional directions for $\mathrm{AD}$ research in 2013 and beyond. Future AD therapies may well, in part, involve probiotic approaches, especially as a prophylactic tactic before mild-cognitive impairment (MCI) or AD is first diagnosed. The implications of altered host-HM interactions in neurological disease would be farreaching indeed, and these may engender novel microbiome manipulative strategies, tailored to the host, for the more effective therapeutic management of $\mathrm{AD}$ and related neuropsychiatric disorders.

\section{ACKNOWLEDGMENTS}

This work was presented in part at the 42nd Society for Neuroscience Meeting, New Orleans LA, October 2012. Research in the Lukiw laboratory involving miRNA, the innate-immune response, amyloidogenesis and neuro-inflammation in $\mathrm{AD}$ and prion disease, was supported through a COBRE Project, a Translational Research Initiative Grant (LSUHSC), the Louisiana Biotechnology Research Network, Alzheimer Association Investigator-Initiated Research Grant IIRG-09-131729, and NIA Grants AG18031 and AG038834.

\section{REFERENCES}

Alexandrov, P. N., Dua, P., Hill, J. M., Bhattacharjee, S., Zhao, Y., and Lukiw, W. J. (2012). microRNA (miRNA) speciation in Alzheimer's disease (AD) cerebrospinal fluid (CSF) and extracellular fluid (ECF). Int. J. Biochem. Mol. Biol. 3, 365-373.

Aziz, Q., Doré, J., Emmanuel, A., Guarner, F., and Quigley, E. M. (2013). Gut microbiota and gastrointestinal health: current concepts and future directions. Neurogastroenterol. Motil. 25, 4-15. doi: 10.1111/nmo.12046

Ball, M. J., Lukiw, W. J., Kammerman, E. M., and Hill, J. M. (2013). Intracerebral propagation of Alzheimer's disease: strengthening evidence of a herpes simplex virus etiology. Alzheimers Dement. 9, 169-175. doi: 10.1016/j.jalz.2012.07.005
Bravo, J. A., Julio-Pieper, M., Forsythe, P., Kunze, W., Dinan, T. G., Bienenstock, J., et al. (2012). Communication between gastrointestinal bacteria and the nervous system. Curr. Opin. Pharmacol. 12, 667-672. doi: 10.1016/j.coph.2012.09.010

Brenner, S. R. (2013). Blue-green algae or cyanobacteria in the intestinal micro-flora my produce neurotoxins such as Beta-N-Methylamino-L-Alanine (BMAA) which may be related to development of amyotrophic lateral sclerosis, Alzheimer's disease and Parkinsons-Dementia-Complex in humans and Equine Motor Neuron Disease in horses. Med. Hypotheses 80, 103-108. doi:10.1016/j.mehy.2012. 10.010

Buryachkovskaya, L., Sumarokov, A., and Lomakin, N. (2013). Historical overview of studies on inflammation in Russia. Inflamm. Res. 62, 441-450. doi: 10.1007/s00011-013-0597-0

Camfield, D. A., Owen, L., Scholey, A. B., Pipingas, A., and Stough, C. (2011). Dairy constituents and neurocognitive health in ageing. Br. J. Nutr. 106, 159-174. doi: 10.1017/S0007114511000158

Carlino, D., De Vanna, M., and Tongiorgi, E. (2013). Is altered BDNF biosynthesis a general feature in patients with cognitive dysfunction? Neuroscientist 19, 345-353. doi: 10.1177/1073858412469444

Carrasco-Pozo, C., Mizgier, M. L., Speisky, H., and Gotteland, M. (2012). Differential protective effects of quercetin, resveratrol, rutin and epigallocatechin gallate against mitochondrial dysfunction induced by indomethacin. Chem. Biol. Interact. 195, 199-205. doi: 10.1016/j.cbi. 2011.12.007

Collins, S. M., Kassam, Z., and Bercik, P. (2013). The adoptive transfer of behavioral phenotype via the intestinal microbiota: experimental evidence and clinical implications. Curr. Opin. Microbiol. 16, 240-245. doi: 10.1016/j.mib.2013.06.004

Douglas-Escobar, M., Elliott, E., and Neu, J. (2013). Effect of intestinal microbial ecology on the developing brain. JAMA Pediatr. 167, 374-379. doi: 10.1001/jamapediatrics.2013.497

Duncan, S. H., and Flint, H. J. (2013). Probiotics and prebiotics and health in ageing populations. Maturitas 75, 44-50. doi: 10.1016/j.maturitas.2013.02.004

Forsythe, P., Kunze, W. A., and Bienenstock, J. (2012). On communication between gut microbes and the brain. Curr. Opin. Gastroenterol. 28, 557-562. doi: 10.1097/MOG.0b013e3283572ffa

Foster, J. A., and McVey Neufeld, K. A. (2013). Gutbrain axis: how the microbiome influences anxiety and depression. Trends Neurosci. 36, 305-312. doi: 10.1016/j.tins.2013.01.005

Hayashi, M. (2013). Anti-basal ganglia antibody. Brain Nerve. 65, 377-384.

He, F., and Balling, R. (2013). The role of regulatory $\mathrm{T}$ cells in neurodegenerative diseases. Wiley Interdiscip Rev. Syst. Biol. Med. 5, 153-180. doi: 10.1002/wsbm.1187

Heijtz, R. D., Wang, S., Anuar, F., Qian, Y., Björkholm, B., Samuelsson, A., et al. (2011). Normal gut microbiota modulates brain development and behavior. Proc. Natl. Acad. Sci. U.S.A. 108, 3047-3052. doi: 10.1073/pnas.1010529108

Hill, J. M., Zhao, Y., Clement, C., Neumann, D. M., and Lukiw, W. J. (2009). HSV1 infection of human brain cells induces miRNA-146a and Alzheimer-type inflammatory 
signaling. Neuroreport 20, 1500-1505. doi: 10.1097/WNR.0b013e3283329c05

Hornig, M. (2013). The role of microbes and autoimmunity in the pathogenesis of neuropsychiatric illness. Curr. Opin. Rheumatol. 25, 488-795. doi: 10.1097/BOR.0b013e32836208de

Lakhan, S. E., Caro, M., and Hadzimichalis, N. (2013). NMDA receptor activity in neuropsychiatric disorders. Front. Psychiatry 4, 52-55. doi: 10.3389/fpsyt.2013.00052

Lu, B., Nagappan, G., Guan, X., Nathan, P. J., and Wren, P. (2013). BDNF-based synaptic repair as a disease-modifying strategy for neurodegenerative diseases. Nat Rev Neurosci. 14, 401-416. doi: $10.1038 / \mathrm{nrn} 3505$

Lukiw, W. J. (2013). Variability in micro RNA (miRNA) abundance, speciation and complexity amongst different human populations and potential relevance to Alzheimer's disease (AD). Front. Cell. Neurosci. 7:133. doi: 10.3389/fncel. 2013.00133

Lukiw, W. J., and Bazan, N. G. (2006). Survival signaling in Alzheimer's disease. Biochem. Soc. Trans. 34, 1277-1282.

Manuelidis, L. (2013). Infectious particles, stress, and induced prion amyloids: A unifying perspective. Virulence 4, 373-383. doi: 10.4161/viru.24838

McVey Neufeld, K. A., Mao, Y. K., Bienenstock, J., Foster, J. A., and Kunze, W. A. (2013). The microbiome is essential for normal gut intrinsic primary afferent neuron excitability in the mouse. Neurogastroenterol. Motil. 25, 183-188. doi: $10.1111 /$ nmo. 12049

Mitew, S., Kirkcaldie, M. T., Dickson, T. C., and Vickers, J. C. (2013). Altered synapses and gliotransmission in Alzheimer's disease and $\mathrm{AD}$ model mice. Neurobiol. Aging 34, 2341-2351. doi: 10.1016/j.neurobiolaging.2013.04.010

Mulligan, V. K., and Chakrabartty, A. (2013). Protein misfolding in the late-onset neurodegenerative diseases: common themes and the unique case of amyotrophic lateral sclerosis. Proteins 81, 1285-1303. doi: 10.1002/prot.24285
Paula-Lima, A. C., Brito-Moreira, J., and Ferreira, S. T. (2013). Deregulation of excitatory neurotransmission underlying synapse failure in Alzheimer's disease. J. Neurochem. 126, 191-202. doi: 10.1111/jnc.12304

Prasad, K. M., Watson, A. M., Dickerson, F. B., Yolken, R. H., and Nimgaonkar, V. L. (2012). Exposure to herpes simplex virus type 1 and cognitive impairments in individuals with schizophrenia. Schizophr. Bull. 38, 1137-1148. doi: 10.1093/schbul/ sbs046

Reijerkerk, A., Lopez-Ramirez, M. A., van Het Hof, B., Drexhage, J. A., Kamphuis, W. W., Kooij, G. et al. (2013). MicroRNAs regulate human brain endothelial cell-barrier function in inflammation: implications for multiple sclerosis. J. Neurosci. 17, 6857-6863. doi: 10.1523/JNEUROSCI.396512.2013

Sarkies, P., and Miska, E. A. (2013). Molecular biology. Is there social RNA? Science 341, 467-468. doi: 10.1126/science.1243175

Saulnier, D. M., Ringel, Y., Heyman, M. B., Foster, J. A., Bercik, P., Shulman, R. J., et al. (2013). The intestinal microbiome, probiotics and prebiotics in neurogastroenterology. Gut Microbes 4, 17-27. doi: 10.4161/gmic. 22973

Schwartz, K., and Boles, B. R. (2013). Microbial amyloids-functions and interactions within the host. Curr. Opin. Microbiol. 16, 93-99. doi: 10.1016/j.mib.2012.12.001

Singh, V. P., Sharma, J., Babu, S., Rizwanulla Singla, A (2013). Role of probiotics in health and disease: a review. J. Pak. Med. Assoc. 63, 253-257.

Slashinski, M. J., McCurdy, S. A., Achenbaum, L. S. Whitney, S. N., and McGuire, A. L. (2012). "Snakeoil," "quack medicine," and "industrially cultured organisms:" biovalue and the commercialization of human microbiome research. BMC Med. Ethics. 30, 13-28. doi: 10.1186/1472-6939-13-28

Tran, L., and Greenwood-Van Meerveld, B. (2013). Age-associated remodeling of the intestinal epithelial barrier. J. Gerontol. A Biol. Sci. Med. Sci. 68, 1045-1056. doi: 10.1093/gerona/glt106
Udit, S., and Gautron, L. (2013). Molecular anatomy of the gut-brain axis revealed with transgenic technologies: implications in metabolic research. Front. Neurosci. 7, 134-137. doi: 10.3389/fnins.2013.00134

Venter, J. C., Adams, M. D., Myers, E. W., Li, P. W., Mural, R. J., Sutton, G. G., et al. (2001). The sequence of the human genome. Science 291, 1304-1351. doi: 10.1126/science. 1058040

von Geldern, G., and Mowry, E. M. (2012). The influence of nutritional factors on the prognosis of multiple sclerosis. Nat. Rev. Neurol. 8, 678-689. doi: 10.1038/nrneurol. 2012.194

Xie, G., Zhang, S., Zheng, X., and Jia, W. (2013). Metabolomics approaches for characterizing metabolic interactions between host and its commensal microbes. Electrophoresis. doi: 10.1002/ elps.201300017. [Epub ahead of print].

Zhao, Y., Cui, J. G., and Lukiw, W. J. (2006). Natural secretory products of human neural and microvessel endothelial cells: implications in pathogenic "spreading" and Alzheimer's disease. Mol. Neurobiol. 34, 181-192. doi: 10.1385/ $\mathrm{MN}: 34: 3: 181$

Received: 15 August 2013; accepted: 26 August 2013; published online: 17 September 2013.

Citation: Bhattacharjee S and Lukiw WJ (2013) Alzheimer's disease and the microbiome. Front. Cell. Neurosci. 7:153. doi: 10.3389/fncel.2013.00153

This article was submitted to the journal Frontiers in Cellular Neuroscience.

Copyright (C) 2013 Bhattacharjee and Lukiw. This is an open-access article distributed under the terms of the Creative Commons Attribution License (CC BY). The use, distribution or reproduction in other forums is permitted, provided the original author(s) or licensor are credited and that the original publication in this journal is cited, in accordance with accepted academic practice. No use, distribution or reproduction is permitted which does not comply with these terms. 